\title{
LESÃO IATROGÊNICA DA VIA BILIAR PRINCIPAL EM COLECISTECTOMIA VIDEOLAPAROSCÓPICA
}

\author{
IATROGENIC BILIARY TRACT INJURY DURING LAPAROSCOPIC \\ CHOLECYSTECTOMY
}

Marcel Milcent, ACBC-RJ' ; Elizabeth G. Santos, TCBC-RJ²; Guilherme Pinto Bravo Neto, TCBC-RJ ${ }^{3}$

\begin{abstract}
RESUMO: Objetivo: Estudar a incidência, mortalidade e morbidez da lesão iatrogênica da via biliar em um Hospital Universitário onde os pacientes foram operados por vários cirurgiões em diferentes fases de treinamento (residentes e "staffs"). Método: Estudo retrospectivo de pacientes operados no Hospital Universitário Clementino Fraga Filho da Universidade Federal do Rio de Janeiro (HUCFF-UFRJ) no período entre janeiro de 1992 e dezembro de 2003. Foram pesquisadas as lesões da via biliar principal, o tempo de reconhecimento das mesmas (per ou pós operatória) e o tipo de reparo utilizado. Resultados: Foram estudados 1589 pacientes com índice de lesão da via biliar de $0.25 \%$, as quais ocorreram principalmente nos últimos anos do uso da técnica no hospital. Conclusões: A incidência de lesões da via biliar foi semelhante à da literatura e bastante próxima à da cirurgia convencional, e não esteve diretamente relacionada à curva de aprendizado do cirurgião (Rev. Col. Bras. Cir. 2005; 32(6): 332-336).
\end{abstract}

Descritores: Trato biliar; Doença iatrogênica; Incidência; Colecistectomia.

\section{INTRODUÇÃO}

A via laparoscópica tem sido reconhecida como padrão de excelência para a colecistectomias ${ }^{1}$. Phillipe Mouret foi quem primeiro a realizou em 1987, mas outros procedimentos já haviam sido realizados por laparoscopia e foram descritos por ginecologistas. Desenvolvida no final da década de 80 e início dos anos 90, a videolaparoscopia mudou os conceitos de acesso cirúrgico e campo operatório, introduzindo a concepção de "cirurgia minimamente invasiva”.

Em 1992 realizou-se a primeira colecistectomia videolaparoscópica (CVL) no Hospital Universitário Clementino Fraga Filho (HUCFF) da Universidade Federal do Rio de Janeiro (UFRJ). Os procedimentos eram realizados por um grupo de cirurgiões entusiastas, progressistas e altruístas que permitiram a viabilização e o ensino rotineiro desse método no HUCFF. Com eles vieram , cedidos ou doados ao Hospital, pinças, insufladores, óticas, câmeras e tudo que era necessário para que as próximas gerações de jovens cirurgiões deste Hospital pudessem usufruir do privilégio da moderna tecnologia médica, que já, há tempos, era conhecida em outros países. Este trabalho é dedicado a eles.

A colecistectomia é um dos procedimentos cirúrgicos mais realizados no mundo. Com o advento da videolaparoscopia, tornou-se uma cirurgia menos traumática, mais estética, com períodos mais curtos de internação. Em contrapartida, observou-se o aumento da incidência de lesões de via biliar extra-hepática quando comparado ao procedimento aberto, ${ }^{2-6}$ fato preocupante devido à morbidade elevada desse tipo de lesão, cuja mortalidade não é desprezível $^{7-11}$.

\section{MÉTODO}

Estudo retrospectivo, de análise de prontuários, das colecistectomias por vídeo realizadas no HUCFF-UFRJ num período de doze anos (de janeiro de 1992 a dezembro de 2003), com informações sobre lesões per-operatórias da via biliar principal decorrentes do procedimento.

No protocolo de estudo as lesões foram assim classificadas:

1- lesões da via biliar principal, em qualquer altura (dos ductos hepáticos direito e esquerdo, confluência dos hepáticos, porções média e distal do colédoco), determinando extravazamento de bile ou interrupção do contraste, notados no pér-operatório ou pós-operatório imediato.

2- lesões da via biliar identificadas no pós-operatório tardio determinando icterícia obstrutiva, e confirmadas por exames de imagem e de laboratório.

A soltura do "clip" da ligadura do ducto cístico, ainda que causadora de coleperitôneo pós-operatório, não foi considerada lesão da via biliar principal.

Além dos dados pertinentes à lesão, também foram coletadas as seguintes informações: sexo, idade, data da cirurgia, experiência do cirurgião (avaliado pelo volume de cirurgias videolaparoscópicas já realizadas).

1. Aluno do curso de Mestrado em Cirurgia do Departamento de Cirurgia da Universidade Federal do Rio de Janeiro (UFRJ).

2. Cirurgiã do Serviço de Cirurgia Geral Hospital Universitário Clementino Fraga Filho da UFRJ (HUCFF-UFRJ).

3. Professor Adjunto do Departamento Cirurgia da UFRJ.

Recebido em 30/09/2005

Aceito para publicação em 01/12/2005

Conflito de interesses: nenhum

Fontes de financiamento: nenhuma

Trabalho realizado no Serviço de Cirurgia Geral do HUCFF-UFRJ. 


\section{RESULTADOS}

Foram realizadas 1589 colecistectomias videolaparoscópicas em pacientes adultos, com distribuição demonstrada no Gráfico 1. Quinze por cento (238) dos pacientes operados eram homens, e 85\% (1351) mulheres. A média de idade foi de 48 anos (12 a 95). O tempo médio de internação para o procedimento foi de 1,69 dias (um a 83). Deve-se observar que o tempo de internação não corresponde ao tempo real de pósoperatório já que muitos pacientes ficaram internados vários dias antes da cirurgia. O tempo de seguimento pós-operatório foi, em média, de 279,12 dias (dois a 2541).

Os Gráficos 2 e 3 mostram respectivamente a distribuição das lesões e a variação da incidência ao longo dos anos do estudo. Os quatro casos $(0,25 \%)$ nos quais houve lesão de via biliar são descritos a seguir:

1 - R.V.S., feminina, 34 anos, operada por médico residente, supervisionado por "staff” em campo. Houve dúvida sobre a anatomia após clipagem e secção do suposto ducto cístico. Foi realizada colangiografia, que não contrastou o colédoco. Após conversão foi evidenciada secção completa do ducto colédoco em sua porção médio-proximal. Procedeuse à anastomose término-terminal moldada sobre o dreno de Kehr. O dreno foi fechado no oitavo dia pós-operatório e a paciente recebeu alta hospitalar com dreno no dia seguinte. Após três meses de acompanhamento ambulatorial, o dreno foi retirado, tendo a paciente recebido alta ambulatorial oito meses depois, com ultra-sonografia de vias biliares normal

2 - M.S.B., feminina, 47 anos, operada por "staff" do serviço de cirurgia. Colangiografia não contrastou o colédoco, mostrando apenas o ducto biliar principal direito. Os "clips" foram retirados e a cirurgia convertida por suspeita de lesão de colédoco. Na conversão foi identificada vesícula séssil implantada no ducto hepático direito, com lesão local. Foi realizada rafia da lesão com dreno de Kehr exteriorizado pela sutura. O dreno foi fechado no $13^{\circ}$ dia pós-operatório e a paciente recebeu alta no dia seguinte. Seis meses depois o dreno foi retirado, e a paciente permanece assintomática vinte meses após a cirurgia.

3 - M.M.J., masculino, 79 anos, operado por "staff" do serviço de cirurgia. Cirurgia sem relato de dificuldades . Reoperado 72 horas depois por abdômen agudo, tendo sido evidenciado coleperitôneo e lesão no ducto hepático direito. Colocado dreno de Kehr n${ }^{\circ} 14$ no hepático direito. Dez dias depois foi reoperado por evisceração, permanecendo em unidade de terapia intensiva por 38 dias e semi-intensiva por mais 13 dias. Recebeu alta hospitalar dois meses e 22 dias após a primeira cirurgia, não havendo relato sobre o fechamento ou a retirada do dreno no prontuário, bem como de qualquer consulta pós-alta.

4 - A.M.M., feminina, 80 anos, operada por "staff" do serviço de cirurgia sem relato de dificuldade durante o procedimento. Reoperada 96 horas depois por coleperitôneo. Encontrada seç̧ão do ducto hepático comum ao nível da confluência dos hepáticos. Realizada hepático-jejunostomia em $\mathrm{Y}$ de Roux, com dreno de Kehr trans-anastomótico. Permaneceu em unidade semi-intensiva por 12 dias e teve o dreno fechado no $15^{\circ}$ dia de pós-operatório. Evoluiu com fístula biliar

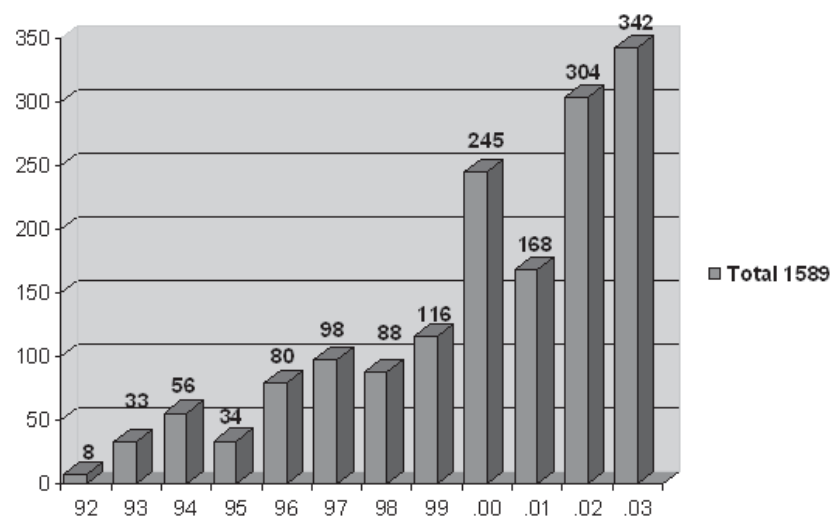

Gráfico 1 - Distribuição dos procedimentos realizados ao longo dos anos.

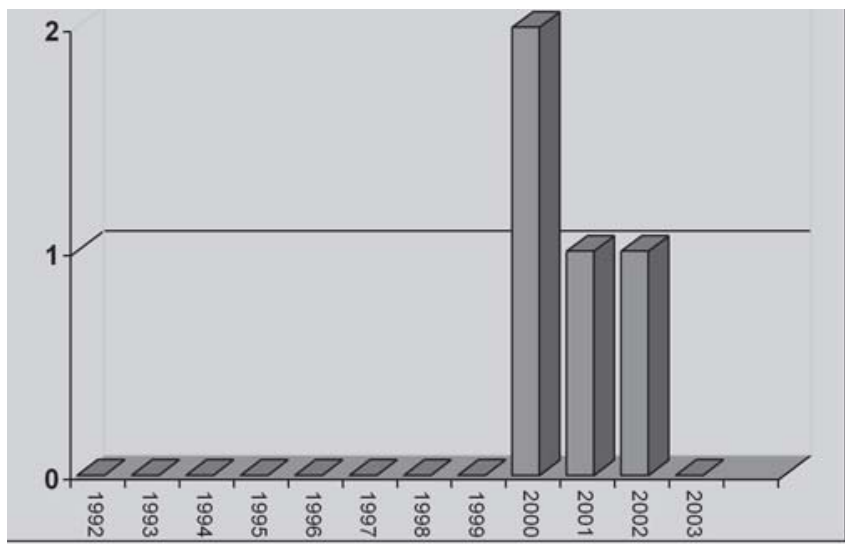

Gráfico 2 - Distribuição das lesões ao longo dos anos.

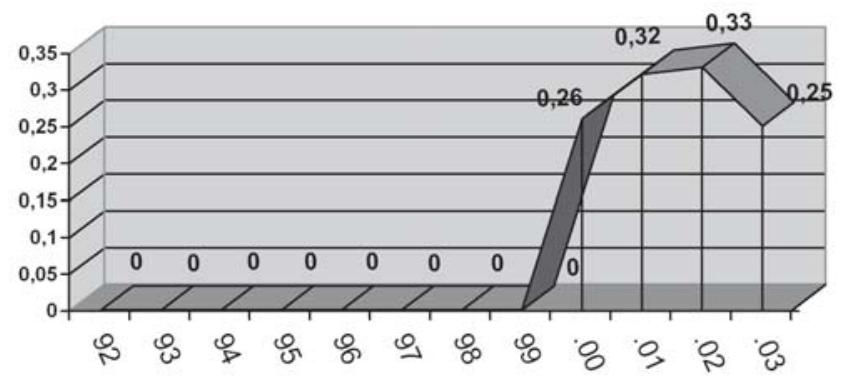

Gráfico 3 - Variação da incidência de lesões ao longo dos anos.

orientada para a pele, que recebeu tratamento conservador, e obteve alta 55 dias após a primeira cirurgia. Foi reinternada 21 dias depois da alta por reabertura da fístula, associada a coleção sub-hepática, que foi drenada. Recebeu alta um mês depois, e encontrava-se assintomática , oito meses depois.

\section{DISCUSSÃO}

Alguns autores tentaram identificar fatores de risco para lesões da via biliar em colecistectomias videolaparoscópicas ${ }^{12-14}$. Foram responsabilizados: 1 - pouca experiência do cirurgião, inclusive com o procedimento convencional (curva de aprendizado); 2 - condições locais desfavoráveis como vesícula esclero-atrófica, aderências perivesiculares, colecistite aguda, variações anatômicas 3 - tempo operatório prolongado (provavelmente relacionado aos outros dois fatores). 
Em relação à curva de aprendizado, diz-se que a lesão da via biliar sofre diminuição progressiva com o passar do tempo, atingindo valor comparável ao dos procedimentos abertos (em torno de $0,3 \%)^{2,4,5}$. A esse fato atribui-se a evolução técnica dos cirurgiões, que estariam num ponto mais alto da curva de aprendizado $^{14,15}$. Paulatinamente, casos antes considerados inadequados para o método laparoscópico começaram a ser tratados com sucesso por vídeo. Grande exemplo é a colecistite aguda, que inicialmente era considerada contra-indicação para o método e hoje, na maioria das vezes, é assim resolvida ${ }^{13,16-18}$.

Já se sabe que o cirurgião mais experiente em cirurgia videolaparoscópica tem menores chances de provocar lesões da via biliar durante CVLs, assim como em qualquer outra cirurgia. Há evidências, contudo, que indicam um novo pico na incidência de lesões da via biliar entre cirurgiões com mais de 100 procedimentos, provavelmente por tenderem a operar casos mais difíceis com o passar do tempo e talvez por se tornarem mais ousados ${ }^{4}$.

Outro aspecto a ser considerado são as variações anatômicas da via biliar, às quais o cirurgião deve sempre estar atento ${ }^{19,20}$. Um ducto cístico curto, vesícula séssil drenando diretamente no ducto hepático comum ou hepático direito, podem gerar interpretações errôneas quanto à identificação do ducto cístico, tornando sua individualização , fundamental na cirurgia, por vêzes impossível.

Desde o início da era videolaparoscópica o uso da colangiografia per-operatória de rotina tem sido questionado. Seu uso rotineiro diminuiria a incidência de lesões da via biliar durante CVLs ${ }^{21}$ ? Os que são contrários à colangiografia peroperatória de rotina argumentam que sua adoção não diminuiu o número de lesões, e que tal conduta aumenta os custos do procedimento e o tempo operatório ${ }^{22}$. Já os adeptos de seu uso rotineiro atestam que o tempo operatório não é substancialmente afetado com uma equipe treinada, e que os custos diretos e indiretos decorrentes de uma única lesão de via biliar ultrapassam, em muito, os de todas as colangiografias realizadas ao longo de anos em uma instituição ${ }^{23}$.

Não se pode negar que a colangiografia per-operatória é útil no reconhecimento da anatomia das vias biliares, e que, ainda que não evite a ocorrência da lesão, ajuda na sua detecção ainda no período intra-operatório ${ }^{24,25}$, o que pode diminuir o grande impacto na morbidade e na qualidade de vida que tais lesões representam para os pacientes. Ligaduras, secções completas ou parciais da via biliar e a visualização de extravazamentos de bile, o que muitas vezes é difícil devido ao pequeno tamanho da lesão, podem passar despercebidos sem a colangiografia.

Não percebida no momento da cirurgia, a lesão pode ser diagnosticada sob várias formas, que guardam relação com o tipo de sintomatologia apresentada: 1 - coleperitônio: sepse abdominal pós-operatória necessitando reoperação; 2 -

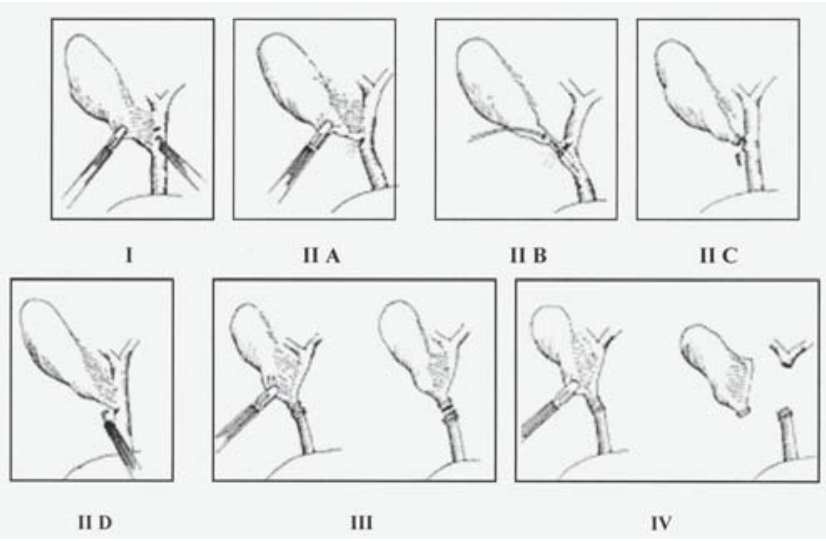

Figura 1 - Amostra esquemática da classificação de Csendes.

fístula biliar: saída de bile por orifício de dreno ou por feridas dos trocáteres; 3 - icterícia precoce ou tardia, com a lesão biliar diagnosticada por colangiopancreatografia, colangiorressonância ${ }^{26}$ ou colangiografia percutânea. A ultrasonografia e a tomografia computadorizada, mostram dilatação de vias biliares intra-hepáticas em vigência de icterícia obstrutiva após colecistectomia videolaparoscópica e sugerem fortemente o diagnóstico, ainda que não o confirmem.

Várias classificações de lesões benignas da via biliar já foram propostas. A classificação de Bismuth ${ }^{27}$ baseia-se na altura da lesão (o ponto onde se acha mucosa biliar saudável) em relação à confluência dos hepáticos. É a classificação mais usada para estenoses tardias, as quais seriam decorrentes, em sua maioria, de lesões térmicas ou de ligaduras muito próximas à via biliar, com reação inflamatória e subseqüente estenose.Não engloba lesões agudas.

A classificação de Strasberg ${ }^{28}$ engloba a classificação de Bismuth (tipos E1 a E5), acrescentando vazamentos de bile decorrentes de lesões do leito vesicular e os decorrentes de soltura de ligadura do cístico, privilegia as lesões decorrentes de variações anatômicas, destinando a elas três tipos (A, B e C), com apenas o tipo D representando lesão da via biliar principal, sem distinção da altura de ocorrência da lesão.

No que tange às lesões agudas, preferimos a classificação de Csendes ${ }^{3}$ que correlaciona melhor o mecanismo de lesão, com sua localização, e proposta terapêutica posterior, não levando em conta as estenoses tardias. Não há referência à soltura do "clip" ou da ligadura do ducto cístico, importante causa de coleperitôneo,tendo em vista que não se trata de lesão da via biliar principal (Tabela 1 e Figura 1).

Vários aspectos técnicos devem ser observados na prevenção de lesões da via biliar em CVLs ${ }^{4}$. Uma técnica de dissecção meticulosa é mandatória, no intuito de identificar o

Tabela 1 - Classificação de Csendes.

\begin{tabular}{ll}
\hline Tipo & Descrição \\
\hline I & Pequena laceração no ducto hepático comum ou hepático direito por eletrocautério ou tesoura \\
II & Lesão da junção cístico-coledociana (tração, eletrocautério, cateter) \\
III & Secção parcial ou completa da via biliar principal \\
IV & Ressecção de mais de $10 \mathrm{~mm}$ da via biliar principal \\
\hline
\end{tabular}


triângulo de Calot, incluindo a vesícula, o ducto cístico, o ducto hepático e a junção dos ductos comuns. A implantação do ducto cístico na vesícula deve ser bem determinada, e o triângulo de Calot, inteiramente dissecado, e aberto de modo que se observe somente o parênquima hepático no assoalho do triângulo, para que nenhuma outra estrutura importante possa ser comprometida .

A dissecção displicente dessa área pode levar a confusão na identificação do ducto cístico e do colédoco, facilitando a ocorrência da clássica “lesão em tenda”, onde se secciona parte da parede lateral do colédoco, por se fazer uma tração cefálica excessiva do cístico. Esta dissecção é dificultada em vários graus nos casos em que há reação inflamatória intensa e/ou um ducto cístico muito curto. O índice de lesões sobe para aproximadamente 3\% nos casos de vesícula escleroatrófica onde tais condições estão geralmente presentes e muitas vezes de forma acentuada ${ }^{28-30}$.

Na ausência de um ducto cístico longo, o mais prudente é proceder à ligadura na altura do infundíbulo vesicular, deixando uma pequena porção deste, sem risco de comprometimento da via biliar principal.

A terapêutica das lesões da via biliar após colecistectomias videolaparoscópicas pode ser dividida em precoce e tardia. Entende-se por terapêutica precoce a que é instituída no mesmo tempo cirúrgico em que se deu a lesão. A colangiografia ${ }^{24,25}$, ainda que não seja adotada de rotina ${ }^{21,22}$, deve ser realizada ao menor sinal de dificuldade.

Lacerações parciais da via biliar em qualquer segmento devem ser tratadas sobre dreno de Kehr ${ }^{3}$. Secções totais ou ressecções com perda de até de $10 \mathrm{~mm}$ de via biliar podem ser tratadas com anastomose término-términal sobre Kehr ou com hepático-jejunostomia ${ }^{10}$, dependendo da preferência e experiência do cirurgião. Lesões com perda de mais de 10 mm devem ser reparadas com hepático-jejunostomia alta.

Os pacientes com lesões identificadas em reoperações de urgência (pós-operatório recente) devem ter sua condição infecciosa sanada e a via biliar drenada, reservando-se o tratamento definitivo para depois ${ }^{27,31}$. Conforme o paciente se apresente clinicamente, pode ser tentada reoperação por videolaparoscopia ${ }^{32}$.

Fístulas bem orientadas decorrentes de vazamento do côto do ducto cístico ou de ductos aberrantes têm boa resposta com a papilotomia endoscópica ${ }^{33,34}$.

As estenoses tardias, produzidas por lesão térmica ou isquêmica, devem ser investigadas quanto à altura em relação à confluência dos hepáticos (classificação de Bismuth), o que definirá a conduta cirúrgica. As lesões Bismuth II e III, e em nossa opinião, as lesões Bismuth I também, devem ser tratadas por hepático-jejunostomia alta, com prolongamento ao ducto hepático esquerdo (procedimento de Hepp-Couinaud ). Já para as do tipo IV e V ficam reservadas as anastomoses intrahepáticas em, freqüentemente, mais de uma cirurgia. ${ }^{27}$

Dependendo da altura da lesão ou existência de acometimentos regionais por estenoses setoriais, ressecções hepáticas podem ser tentadas com a desvatagem de acrescentar maior morbidade para o paciente.

O impacto causado por lesões da via biliar em colecistectomias videolaparoscópicas já foi bem demonstrado. Os casos em que se consegue o reparo primário (no mesmo tempo cirúrgico), ainda que obviamente com maior morbidade e custo do que quando não há lesão, são muito menos onerosos tanto para o paciente quanto para a instituição, que os casos em que o reparo é tardio. O desenvolvimento de estenoses ou fístulas não raro requer várias internações por colangite, o que obriga o paciente a ser submetido a procedimentos invasivos ${ }^{33-35}$ (CPER,dilatações percutâneas), cirurgia para anastomoses biliodigestivas ${ }^{36}$ (frequentemente mais de um procedimento), e ainda assim pode evoluir para cirrose biliar secundária com todas as suas complicações, podendo chegar até mesmo ao transplante hepático ${ }^{37}$.

Em nossa casuística observamos incidência de lesão da via biliar similar à literatura, corroborando a segurança do procedimento, sem mencionar suas já consagradas vantagens.

Pela a distribuição mais concentrada do volume de cirurgias no término do período do estudo, e por 75 \% delas terem ocorrido em operações conduzidas por cirurgiões com pelo menos 50 procedimentos realizados, concluímos que não houve interferência da curva de aprendizado na ocorrência de tais lesões.

Não houve mortalidade decorrente das lesões. Contudo, a morbidade mostrou-se elevada nos pacientes em que a lesão não foi descoberta no próprio procedimento e, conseqüentemente, não corrigida imediatamente. Naturalmente, nesses dois pacientes, houve longa permanência hospitalar, com internação em unidades fechadas, e aumento exponencial dos custos hospitalares.

A realização, em dois pacientes, da colangiografia per-operatória com diagnóstico das lesões, que foram reparadas no mesmo ato operatório, confirma que a colangiografia, embora não evite essas lesões pode diminuir a morbidade da complicação por permitir seu reparo imediato.

Não foram evidenciadas lesões tardias, tais como estenoses por dano térmico. Contudo, o curto tempo de seguimento pós-operatório pode ter comprometido nossa avaliação desse tipo de lesão.

\section{ABSTRACT}

Background: The aim of this study was to analyze the incidence, morbidity and mortality of iatrogenic biliary tract injury during laparoscopic cholecystectomies in a teaching hospital. Methods: Retrospective study at Hospital Universitário Clementino Fraga Filho of the Universidade Federal do Rio de Janeiro from January 1992 to December 2003. Total and partial injuries, the time to recognize them and types of repair were reviewed. Results: During the studied period, 1589 patients underwent laparoscopic cholecystectomies with four biliary tract injuries (0.25\%), all of them occuring in the recent years. Conclusions: The incidence of iatrogenic biliary tract injury in our series was similar to the literature and very similar to the open procedure.

Key words: Biliary tract; Iatrogenic disease; Incidence; Cholecystectomy. 


\section{REFERÊNCIAS}

01. Soper NJ, Stockmann PT, Dunnegan DL, et al. Laparoscopic cholecystectomy. The new "gold standard"? Arch Surg.1992;127(8):917-21; discussion 921-3.

02. Adamsen S, Hansen OH, Funch-Jensen P, et al. Bile duct injury during laparoscopic cholecystectomy: a prospective nationwide series. J Am Coll Surg. 1997;184(6):571-8.

03. Csendes A, Navarrete C, Burdiles P, et al. Treatment of common bile duct injuries during laparoscopic cholecystectomy: endoscopic and surgical management. World J Surg. 2001;25(10):1346-51.

04. Krähenbühl L, Sclabas G, Wente MN, et al. Incidence, risk factors, and prevention of biliary tract injuries during laparoscopic cholecystectomy in Switzerland. World J Surg. 2001;25(10): 1325-30.

05. Richardson MC, Bell G, Fullarton GM. Incidence and nature of bile duct injuries following laparoscopic cholecystectomy: an audit of 5913 cases. West of Scotland Laparoscopic Cholecystectomy Audit Group. Br J Surg. 1996;83(10):1356-60.

06. Way LW, Stewart L, Gantert W, et al. Causes and prevention of laparoscopic bile duct injuries: analysis of 252 cases from a human factors and cognitive psychology perspective. Ann Surg. 2003;237(4):460-9.

07. Al-Ghnaniem R, Benjamin IS. Long-term outcome of hepaticojejunostomy with routine access loop formation following iatrogenic bile duct injury. Br J Surg. 2002;89(9):1118-24.

08. Gouma DJ, Obertop H. Quality of life after repair of bile duct injury. Br J Surg. 2002;89(4):385-6

09. Melton GB, Lillemoe KD, Cameron JL, et al. Major bile duct injuries associated with laparoscopic cholecystectomy: effect of surgical repair on quality of life. Ann Surg. 2002;235(6):88-95

10. Moraca RJ, Lee FT, Ryan JA, et al. Long-term biliary function after reconstruction of major bile duct injuries with hepaticoduodenostomy or hepaticojejunostomy. Arch Surg. 2002;137(8):889-93; discussion 893-4.

11. Savader SJ, Lillemoe KD, Prescott CA, et al. Laparoscopic cholecystectomy-related bile duct injuries:a health and financial disaster. Ann Surg. 1997;225(3):268-73.

12. Andren-Sandberg A, Alinder G, Bengmark S. Accidental lesions of the common bile duct at cholecystectomy. Pre- and perioperative factors of importance. Ann Surg. 1985;201(3):328-32.

13. Kum CK, Eypasch E, Lefering R, et al. Laparoscopic cholecystectomy for acute cholecystitis: is it really safe? World J Surg.1996;20(1):43- 8; discussion 48-9.

14. Moore MJ, Bennett CL. The learning curve for laparoscopic cholecystectomy. The Southern Surgeons Club. Am J Surg.1995;170(1):55-9.

15. Voitk AJ, Tsao SG, Ignatius S. The tail of the learning curve for laparoscopic cholecystectomy. Am J Surg. 2001;182(3):250-3.

16. Eldar S, Sabo E, Nash E, et al. Laparoscopic cholecystectomy for acute cholecystitis: prospective trial. World J Surg. 1997;21(5):540-5

17. Lo CM, Liu CL, Fan ST, et al. Prospective randomized study of early versus delayed laparoscopic cholecystectomyfor acute cholecystitis. Ann Surg. 1998;227(4):461-7

18. Navez B, Mutter D, Russier Y, et al. Safety of laparoscopic approach for acute cholecystitis: retrospective study of 609 cases. World J Surg. 2001;25(10):1352-6.

19. Blumgart LH. Benign biliary structures. In: Surgery of the liver and biliary tract. London: Churchill Livingstone; 1994. pp. 86594.

20. Meyers WC, Peterseim DS, Pappas TN, et al. Low insertion of hepatic segmental duct VII-VIII is an important cause of major biliary injury or misdiagnosis. Am J Surg. 1996;171(1):187-91.
21. Metcalfe MS, Ong T, Bruening $\mathrm{MH}$, et al. Is laparoscopic intraoperative cholangiogram a matter of routine? Am J Surg. 2004;187(4):475-81.

22. Clair DG, Carr-Locke DL, Becker JM, et al. Routine cholangiography is not warranted during laparoscopic cholecystectomy. Arch Surg. 1993;128(5):551-4; discussion 554-5.

23. Flum DR, Flowers C, Veenstra DL. A cost-effectiveness analysis of intraoperative cholangiography in the prevention of bile duct injury during laparoscopic cholecystectomy. J Am Coll Surg. 2003;196(3):385-93.

24. Fletcher DR, Hobbs MST, Tan P, et al. Complications of cholecystectomy: risks of the laparoscopic approach and protective effects of operative cholangiography: a populationbased study. Ann Surg. 1999;229(4):449-57.

25. Woods MS, Traverso LW, Kozarek RA, et al. Biliary tract complications of laparoscopic cholecystectomy are detected more frequently with routine intraoperative cholangiography. Surg Endosc. 1995;9(10):1076-80.

26. Coakley FV, Qayyum A. Magnetic resonance cholangiopancreatography. Gastrointest Endosc. 2002;55(7 Suppl):S2-22.

27. Bismuth H, Majno PE. Biliary strictures: classification based on the principles of surgical treatment. World J Surg. 2001;25(10):1241-4.

28. Strasberg SM, Hertl M, Soper NJ. An analysis of the problem of biliary injury during laparoscopic cholecystectomy. J Am Coll Surg. 1995;180(1):101-25.

29. MacFadyen JB, Vecchio R, Ricardo AE, et al. Bile duct injury after laparoscopic cholecystectomy. The United States experience. Surg Endosc. 1998;12(4):315-21.

30. Targarona EM, Marco C, Balague C, et al. How, when, and why bile duct injury occurs? A comparison between open and laparoscopic cholecystectomy. Surg Endosc. 1998;12(4):322-6.

31. Hepp J, Couinaud C. L'abord et l'utilisation du canal hépatique gauche dans les réparations de la voie biliaire principale. Presse Med. 1956;64:947-8.

32. Wills VL, Jorgensen JO, Hunt DR. Role of relaparoscopy in the management of minor bile leakage after laparoscopic cholecystectomy. Br J Surg. 2000;87(2):176-80.

33. Baron TH, Feitoza AB, Nagorney DM. Successful endoscopic treatment of a complete transection of the bile duct complicating laparoscopic cholecystectomy. Gastrointest Endosc. 2003;57(6):765-9.

34. Davids PH, Ringers J, Rauws EA, et al. Bile duct injury after laparoscopic cholecystectomy: the value of endoscopic retrograde cholangiopancreatography. Gut. 1993;34(9):1250-4.

35. Misra S, Melton GB, Geschwind JF, et al. Percutaneous management of bile duct strictures and injuries associated with laparoscopic cholecystectomy: a decade of experience. J Am Coll Surg. 2004;198(2):218-26

36. Biffl WL, Moore EE, Offner PJ, et al. Routine intraoperative laparoscopic ultrasonography with selective cholangiography reduces bile duct complications during laparoscopic cholecystectomy. J Am Coll Surg. 2001;193(3):272-80.

37. Nordin A, Halme L, Mäkisalo H, et al. Management and outcome of major bile duct injuries after laparoscopic cholecystectomy: from therapeutic endoscopy to liver transplantation. Liver Transpl. 2002;8(11):1036-43.

Endereço para correspondência:

Elizabeth G. Santos

Rua Morais e Silva 126/305

Maracanã

20271-031- Rio de Janeiro - RJ

eligsant54@globo.com 